\title{
MR-Artefakte erkennen und vermeiden
}

\section{Detect and avoid MR artifacts}

S. Heiland

\section{Übersicht \\ Einleitung \\ MR-Artefakte}

\section{Zusammenfassung}

In der Magnetresonanztomografie (MRT) können durch gezielte Wahl der Sequenzparameter unterschiedlichste Kontraste erzeugt werden. Dabei haben unterschiedlichste Gewebe- bzw. Substanzparameter Einfluss auf die Signalintensität, beispielsweise die Relaxationszeiten, die Protonendichte, die Diffusion, der relative Sauerstoffgehalt von Blut oder der Blutfluss. Diese multiparametrische Abhängigkeit des Verfahrens ist die Basis des hohen Weichteilkontrasts der MRT, allerdings ist sie auch der Hauptgrund für die hohe Artefaktanfälligkeit der Methode.

In diesem Artikel werden die unterschiedlichen MR-Artefakte beschrieben, dabei wird insbesondere auf die Ursachen und Methoden zur Vermeidung der Artefakte eingegangen.

\section{Einleitung}

Artefaktempfindlichkeit. Die Magnetresonanztomografie (MRT) weist im Vergleich zu anderen radiologischen Verfahren eine deutlich höhere Sensitivität gegenüber Bildartefakten auf (Abb. 1). Dies hängt zum einen damit zusammen, dass das Bildsignal in der MRT von einer Vielzahl physiologischer und physikalischer Parameter des Gewebes abhängt, z. B. von der Protonendichte, den T1- und T2-Relaxationszeiten, der Stärke der Wasserdiffusion, der Temperatur und der Durchblutung. Ferner beeinflussen Sequenztyp und Sequenzparameter wie auch die MR-Hardware (Feldstärke, Homogenität von statischem Magnetfeld und Gradientenfeld) das MR-Signal.

Komplexe Artefaktmuster. Außerdem ist das Artefaktmuster in der MRT sehr komplex und nicht auf einfache Weise der zugrunde liegenden Ursache zuzuordnen. Dies ist darauf zurückzuführen, dass die Ortscodierung in der MRT aufgrund der hohen Wellenlänge nicht direkt über richtungsabhängige Projektionsmessungen, sondern indirekt über Magnetfeldgradienten und inverse Fourier-Transformation des gemessenen Signals realisiert wird. Aufgrund der Komplexität des daraus resultierenden Artefaktmusters ist eine detaillierte physikalisch-technische Kenntnis der Bildentstehung in der MRT zur Identifizierung und Interpretation der Artefakte nötig.

Dennoch ist es bei der Anwendung der MRT in der radiologischen Diagnostik sehr wichtig, die Artefaktmuster zu (er)kennen und zu wissen, wie man sie vermeidet bzw. minimiert. Denn Artefakte können sowohl pathologische Befunde maskieren als auch krankhafte Veränderungen vortäuschen [1].

Abgrenzung von Bildeffekten. Grundsätzlich ist es nicht einfach, zwischen (positiven) Bildeffekten und (negativen) Bildartefakten systematisch zu unterscheiden: Häufig bildete die systematische Erforschung der Ursache von Artefakten den Kondensationskeim zur Entwicklung innovativer MR-Techniken. So führen beispielsweise Feldverzerrungen, die auf lokalen Inhomogenitäten der Suszeptibilität beruhen, einerseits zu Artefakten wie Signalauslöschungen oder Verzeichnungen, andererseits aber auch zu neuen MR-Kontrasten, die Grundlage von funktionellen Verfahren wie der BOLD-Bildgebung (BOLD = ,blood oxygenation level dependent“') oder der Perfusions-MRT und von 


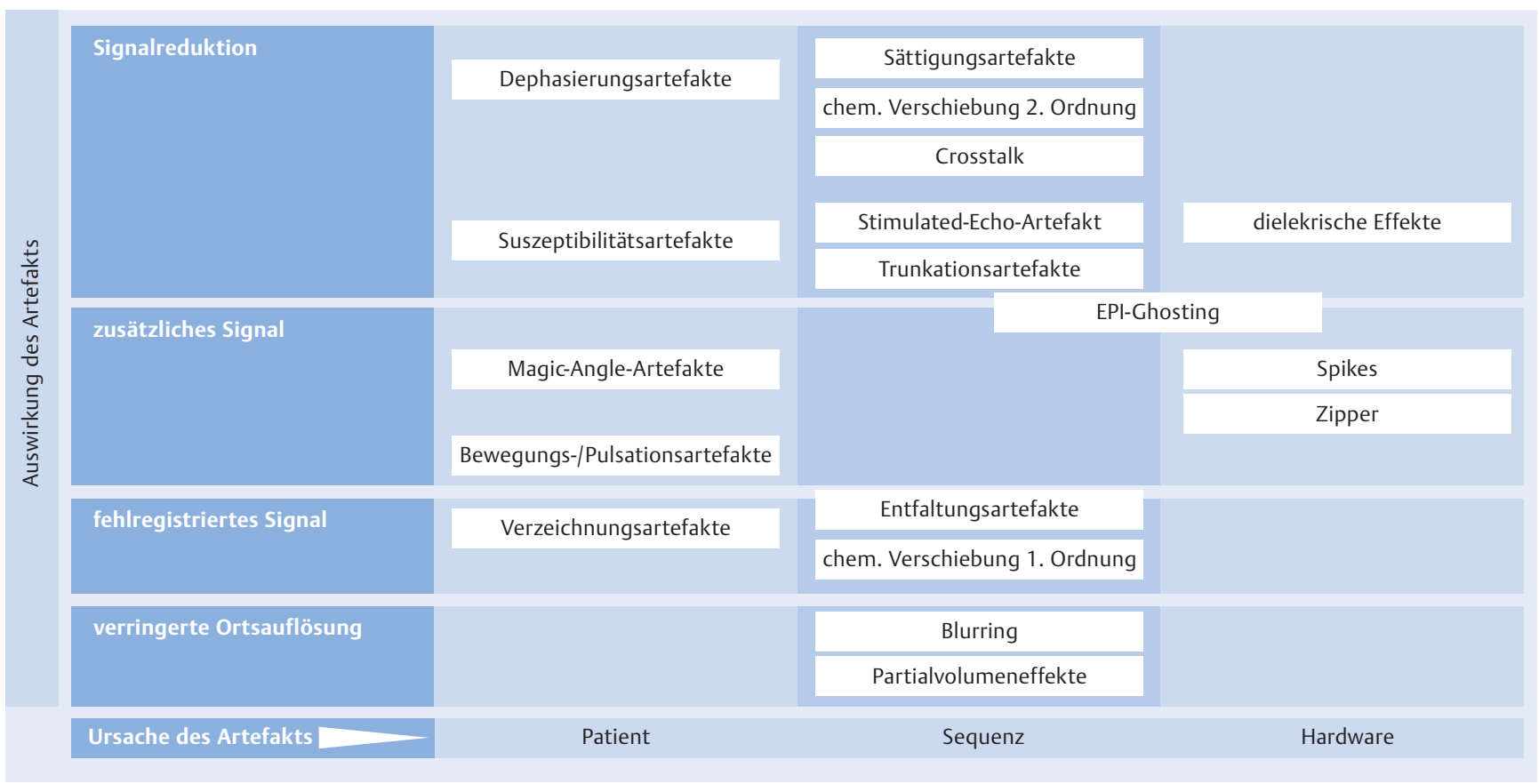

Abb. 1 Systematik der MR-Artefakte: Einordnung nach Artefaktursache und Repräsentation des Artefakts im MR-Bild.

SWI („susceptibility weighted imaging“) sind. Ein anderes Beispiel sind Bewegungsartefakte: Während man grundsätzlich die Bewegungssensitivität von MR-Sequenzen zu minimieren versucht, wurden spezielle Verfahren entwickelt, die auf der Bewegung von Wassermolekülen im Magnetfeld beruhen, so z. B. die Phasenkontrastangiografie und die Diffusions-MRT.

In diesem Artikel werden die wichtigsten MR-Artefakte vorgestellt. Dabei werden für jedes Artefakt die physikalisch-technischen Grundlagen seiner Entstehung erklärt und beschrieben, wie sich diese Mechanismen auf das Bildsignal auswirken. Danach werden Methoden vorgestellt, mit denen die Artefakte minimiert bzw. vermieden werden können.

\section{MR-Artefakte}

\section{Bewegungs- und Pulsationsartefakt}

\section{- Ursache des Artefakts}

Bewegungs- und Pulsationsartefakte sind die häufigsten Artefakte in der MRT. Spins, die sich bewegen, erfahren zwischen Anregungspuls und Datenauslese in Summe ein anderes Gradientenfeld als ruhende Spins. Dies resultiert in Phasenfehlern, die wiederum Ursache für eine Fehlregistrierung im MR-Bild sind.
Zwei unterschiedliche Bewegungstypen können Ursache der Artefakte sein:

- Willkürliche oder unwillkürliche Bewegungen des Körpers oder von Organen (z. B. Darmperistaltik): Die hieraus resultierenden Artefaktmuster sind irregulär und nicht vorhersagbar. Generell bewirken sie verwischte Bilder und Geister außerhalb des bewegten Organs oder Körperteils.

- Pulsatile Strömung von Flüssigkeiten (z. B. fließendes Blut oder Zerebrospinalflüssigkeit): In Phasencodierrichtung resultiert hieraus ein periodisches Muster von Kopien der pulsierenden Struktur. Der Abstand zwischen diesen Geistern ist dabei proportional zur Pulsfrequenz (Abb. 2).

\section{- Vermeidung des Artefakts}

Da Bewegungs- und Pulsationsartefakte unterschiedlichste Ursachen haben können, gibt es unterschiedliche Wege, die Artefakte zu minimieren oder zu vermeiden:

- Reduktion der Bewegung während der MR-Messung: Willkürliche Bewegungen können durch eine stabile Lagerung des untersuchten Körperteils verringert werden. Deutlich schwieriger ist es, unwillkürliche Bewegungen zu vermeiden. Beispielsweise können krampflösende Medikamente verabreicht werden, um die Darmperistaltik einzuschränken.

- Triggerung: Hierbei wird die MR-Messung durch die Erfassung physiologischer Parameter (EKG, Puls, Atembewegung) ausschließlich in definierten Be- 
wegungszuständen durchgeführt, z. B. in einer bestimmten Atemposition oder in einem bestimmten Stadium des Herzzyklus. Allerdings verlängert sich die Akquisitionszeit durch Triggerung i.d.R. erheblich.

- Vorsättigung: Artefakte aufgrund fließenden Bluts können durch eine Vorsättigung der Spins im Blut verringert werden. Hierzu wird entweder auf einer Seite oder auf beiden Seiten des Schichtblocks eine Sättigungsschicht platziert.

- Mittelung: Durch Aufnahme mehrerer Akquisitionen und anschließender Mittelung können vor allem Artefakte aufgrund irregulärer Bewegungen minimiert werden.

- Flusskompensation: Durch spezielle Gradientenschaltungen in einer oder mehreren Gradientenrichtungen wird der Phaseneffekt von linearem oder sinusförmig pulsierendem Blut vermieden [2]. Da hierzu das 1. bzw. 2. Momentum der Gradienten Null sein muss, spricht man auch von „gradient moment nulling“ (GMN). Nachteil dieser Flusskompensationstechniken ist, dass durch die komplexeren Gradientenschaltungen die Echozeit verlängert wird und daher Sequenzen mit ultrakurzen Echozeiten nicht mehr möglich sind.

- Änderung der Phasencodierrichtung: Ist die Artefaktquelle sehr klein und nur ein Teil des Bildes von Interesse, kann ein Wechsel der Phasencodierrichtung sinnvoll sein, falls man hierdurch die Geister aus dem interessierenden Organ bzw. der interessierenden Region hinausdrängen kann.

- Spezielle MR-Sequenzen: Durch Anwendung ultraschneller Sequenzen (EPI, HASTE), die die Bilder innerhalb weniger Millisekunden aufnehmen, kann die Bewegung quasi eingefroren werden. Eine weitere Möglichkeit ist die nachträgliche Bewegungskorrektur durch separate Erfassung der Phaseninformation (Navigator Echo) [3]. Außerdem kann durch radiale Abtastung des k-Raums eine geringere Bewegungssensitivität erreicht werden, da bei dieser Art der Datenaufnahme das Zentrum des k-Raums mehrfach ausgelesen wird [4]. Ein Beispiel für diese Sequenzgruppe ist die PROPELLER-Sequenz (,periodically rotated overlapping parallel lines with enhanced reconstruction“).

\section{Bildunschärfe (Blurring)}

\section{- Ursache des Artefakts}

Werden die hohen Frequenzen im k-Raum nicht aufgenommen, werden Kanten und Grenzflächen anatomischer Strukturen im MR-Bild unscharf dargestellt.

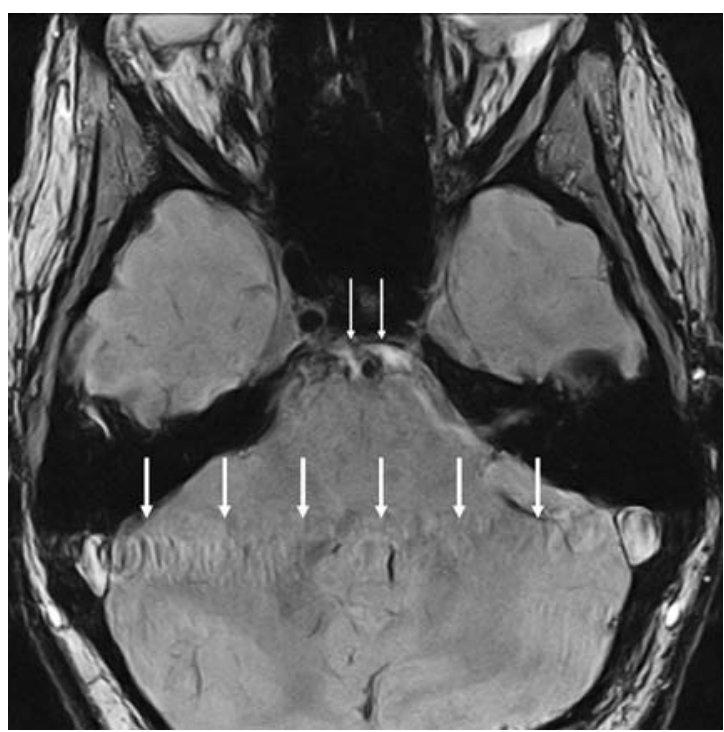

Abb. 2 Flussartefakte: In dieser transversalen MR-Aufnahme sind Flussartefakte des Sinus sigmoideus in Phasencodierrichtung (rechts - links) zu erkennen, außerdem Flussartefakte der A. basilaris.

Dies ist immer dann der Fall, falls die Zahl der Phasencodierschritte, z.B. zur Verkürzung der Messzeit bei dynamischen MR-Sequenzen, reduziert wird. Doch auch bei Wahl einer adäquaten Zahl von Phasencodierschritten kann dieses Artefakt auftreten, falls schnelle Bildgebungstechniken wie Turbo-Spin-Echo-(TSE-) oder EPI-Sequenzen verwendet werden (Abb. 3). Da die Auslesezeit bei diesen Verfahren sehr lang ist, kann es passieren, dass in den äußeren Zeilen des k-Raums nur noch Rauschen akquiriert wird, da aufgrund der hohen Echozeit die Quermagnetisierung bereits zerfallen ist.

\section{- Vermeidung des Artefakts}

Dieses Artefakt kann nur vermieden werden, indem man die Voraussetzungen dafür schafft, dass auch in den äußeren Zeilen des k-Raums Signal erfasst werden kann. Dies kann durch die Wahl des Ausleseschemas geschehen: So ist eine sequenzielle Auslese des k-Raums günstiger als eine zentrische, da bei der sequenziellen Auslese zumindest die Hälfte der hochfrequenten Signalinformationen bei niedrigen Echozeiten aufgenommen wird, während bei der zentrischen nur die Mitte des k-Raums bei niedrigen Echozeiten akquiriert wird, die äußeren Teile des k-Raums jedoch immer bei höheren Echozeiten. Allerdings ist eine zentrische Auslese beispielsweise bei T1- oder PD-gewichteten Sequenzen erforderlich, da hier eine niedrige effektive Echozeit benötigt wird.

Eine zweite Möglichkeit zur Reduktion des Artefakts besteht darin, die Akquisitionszeit für den gesamten Echozug zu verkürzen. Dies ist möglich, indem man die Echozuglänge reduziert, wodurch sich aber Messzeit verlängert. Daher ist hier die Anwendung von Parallel Imaging sinnvoll. Der Echozug kann auch verkürzt 

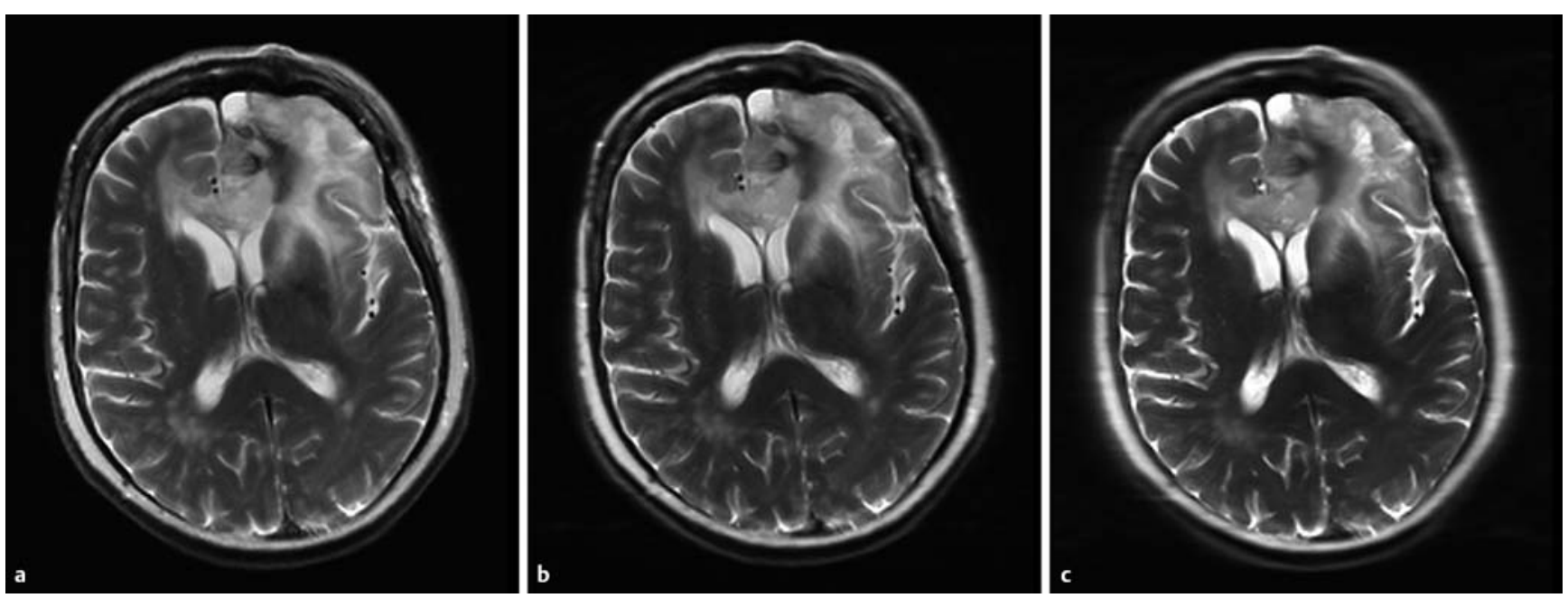

Abb. 3 Transversale TSE-Aufnahmen mit Turbofaktor (a) 9, (b) 17 und (c) 31. Mit zunehmendem Turbofaktor kommt es zu einer Kantenunschärfe (Blurring).

Abb. 4 ChemicalShift-Artefakte 1. Ordnung: In Frequenzcodierrichtung (rechts - links) kommt es an den Grenzen zwischen Fett und Organgewebe zu hypointensen Rändern, jedoch ausschließlich an einer Seite (hier rechts, Pfeile).

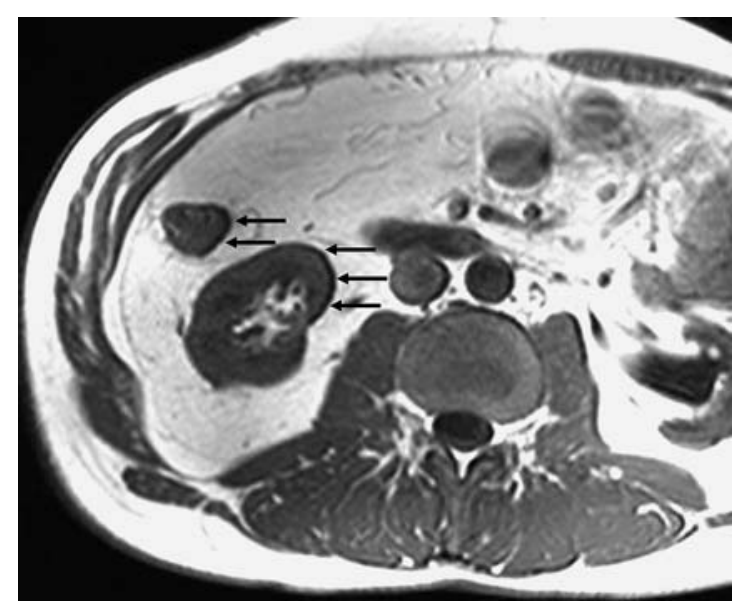

werden, indem die Auslesebandbreite erhöht wird, da hierdurch die Auslesedauer für jedes einzelne Echo verringert wird. Dies ist jedoch nur auf Kosten des Signal-zu-Rausch-Verhältnisses (SNR) möglich.

\section{Chemische Verschiebung 1. Ordnung}

\section{- Ursache des Artefakts}

Auf Wasserstoffprotonen, die in unterschiedlichen Molekülen gebunden sind, wirken wegen der Abschirmungseffekte der Elektronenwolken unterschiedliche effektive Magnetfelder. Daher unterscheiden sich die Präzessionsfrequenzen von Wasser und Fett. Die relative Frequenzverschiebung zwischen dem Wasserpeak und dem Fettpeak beträgt 3,5 ppm. Aufgrund dieser Frequenzverschiebung kommt es während der Datenaufnahme zu einer Dephasierung von Wasser- und Fettprotonen (Abb. 4), die in einer fehlerhaften Registrierung resultiert. Das heißt, Wasser- und Fettproto- nen, die sich am gleichen Ort befinden, werden unterschiedlichen Pixeln zugeordnet. Die Zahl der Pixel, um die Fett- und Wassersignal gegeneinander verschoben sind, ist umso größer, je kleiner die Auslesebandbreite, d.h. je länger die Auslesezeit ist [5]. Bei Standardsequenzen treten Artefakte aufgrund der chemischen Verschiebung ausschließlich in Ausleserichtung auf. Bei EPI-Sequenzen ist dagegen die Bandbreite in Phasencodierrichtung aufgrund des langen Echozugs sehr klein; daher ist der Versatz zwischen Fett- und Wassersignal in EPI-Bildern in Phasencodierrichtung um ein Vielfaches größer als in Standardsequenzen (Abb. 5).

\section{- Vermeidung des Artefakts}

Um die Artefakte aufgrund der chemischen Verschiebung zu minimieren, sollte man die Auslesebandbreite möglichst hoch wählen. Dies führt jedoch aufgrund der kurzen Auslesezeit zu einem geringeren SNR: Da die Bandbreite in Phasencodierrichtung bei EPI-Sequenzen wegen des speziellen Datenausleseschemas nicht wesentlich erhöht werden kann, ist es in diesem Fall unabdingbar, die Fettspins durch einen spektralen Fettsättigungspuls zu unterdrücken (Abb. 5).

\section{Chemische Verschiebung 2. Ordnung}

\section{- Ursache des Artefakts}

Auch dieses Artefakt basieren auf der chemischen Verschiebung zwischen Fett- und Wasserprotonen; im Gegensatz zu den Artefakten 1. Ordnung tritt es jedoch ausschließlich bei Gradienten-Echo-Sequenzen auf. Wie oben beschrieben, führt die unterschiedliche Präzessionsfrequenz von Fett- und Wasserprotonen zu 
einer Phasenverschiebung, die sich mit der Zeit nach der Hochfrequenzanregung verändert. Beträgt diese Phasenverschiebung $180^{\circ}$, wird in Volumenelementen, in denen sowohl Wasser als auch Fett vorhanden ist, das resultierende Signal niedriger, da sich die Magnetisierungskomponenten - abhängig von der relativen Konzentration der Fett- und Wasserprotonen - teilweise oder völlig auslöschen („opposed-phase“-Bedingung) [6]. Daher entstehen bei Gradienten-EchoSequenzen mit der Echozeit

$$
T E_{\text {opposed }}=\frac{2 \mathrm{~N}-1}{2 \cdot 3.5 p p m \cdot \gamma \cdot B_{0}}, \mathrm{~N}=1,2,3, \ldots
$$

dunkle Linien an der Grenzfläche zwischen Fett und anderen Geweben ( $\gamma$ : gyromagnetisches Verhältnis, $\mathrm{B}_{0}$ : statisches Magnetfeld) (Abb. 6). Diese Artefakte sind bei 1,5 Tesla für Echozeiten von z. B. 2,2 ms und 6,6 ms zu sehen.

\section{- Vermeidung des Artefakts}

Dieses Artefakt kann leicht vermieden werden, indem man Echozeiten wählt, in denen die Fett- und Wasserspins in die gleiche Richtung zeigen, da sich dann die Signale der Wasser- und Fettprotonen verstärken. Dies ist der Fall für folgende Echozeiten:

$$
T E_{\text {in }}=\frac{N}{3.5 p p m \cdot \gamma \cdot B_{0}}, \mathrm{~N}=1,2,3, \ldots
$$

Allerdings ist das Artefakt in manchen Fällen diagnostisch von Nutzen, z. B., um fettige Infiltrationen in der Leber sensitiv darstellen zu können [7].

\section{Crosstalk}

\section{- Ursache des Artefakts}

Da die Hochfrequenzpulse in der MR-Bildgebung sehr kurz sind (im Bereich von Mikrosekunden), weicht das Schichtprofil grundsätzlich von der idealen Rechteckfunktion ab. Daher werden auch in benachbarten Schichten Spins angeregt, was wiederum zur partiellen Sättigung bzw. zu einem veränderten Bildkontrast in benachbarten Schichten führt.

\section{Vermeidung des Artefakts}

Dieses Artefakt kann weitgehend vermieden werden, indem man zwischen den Schichten eine Schichtlücke von 10-20\% der Schichtdicke wählt, in der keine Anregung bzw. Auslese erfolgt. Je kürzer der verwendete Hochfrequenzpuls, umso größer ist die Schichtlücke, die gewählt werden muss, um das Artefakt vollständig zu unterdrücken. Allerdings besteht die Gefahr, dass man auf diese Weise sehr kleine Läsionen übersieht,

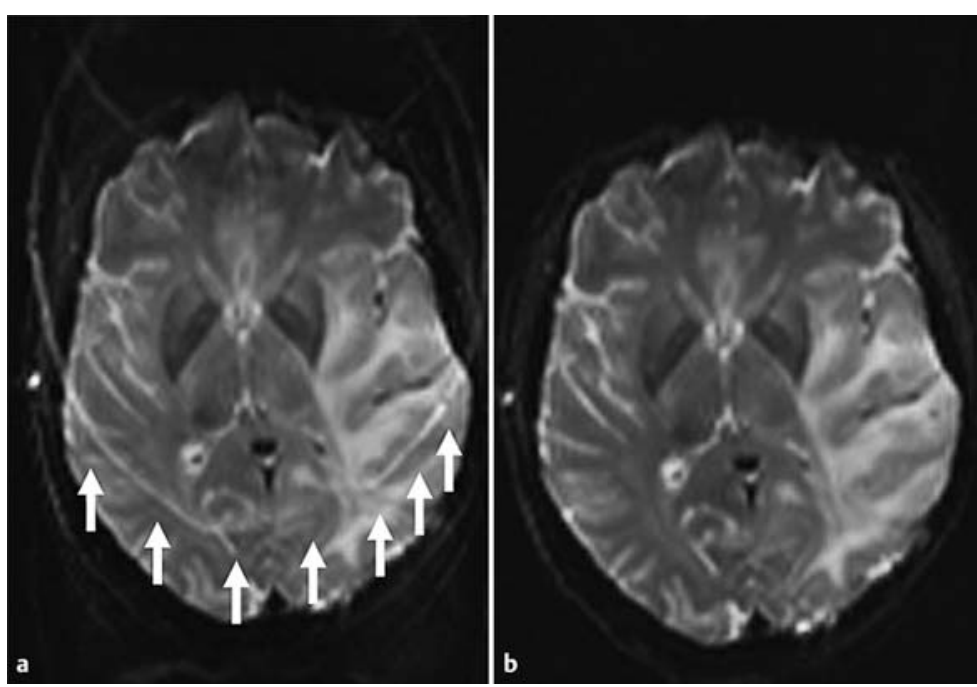

Abb. 5 Spin-Echo-EPI-Sequenz. a Aufgrund der extrem geringen Bandbreite in Phasencodierrichtung (anterior - posterior) sind die Fett- und Wassersignale um mehrere Pixel gegeneinander verschoben (Pfeile). b Durch spektrale Fettsättigung kann dieses Artefakt vermieden werden.

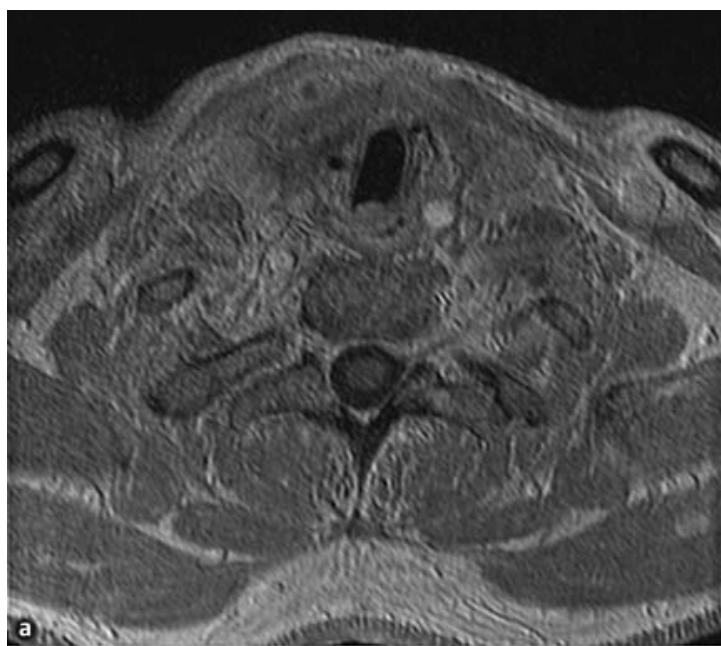

Abb. 6 ChemicalShift-Artefakte 2. Ordnung bei einer GradientenEcho-Sequenz. a TE ist so gewählt, dass Wasser- und Fettprotonen in Phase sind. b Aufnahme in der "opposed-phase“Bedingung. Zwischen Fettgewebe und wasserhaltigen Strukturen kommt es zu hypointensen Rändern. Im Gegensatz zu ChemicalShift-Artefakten

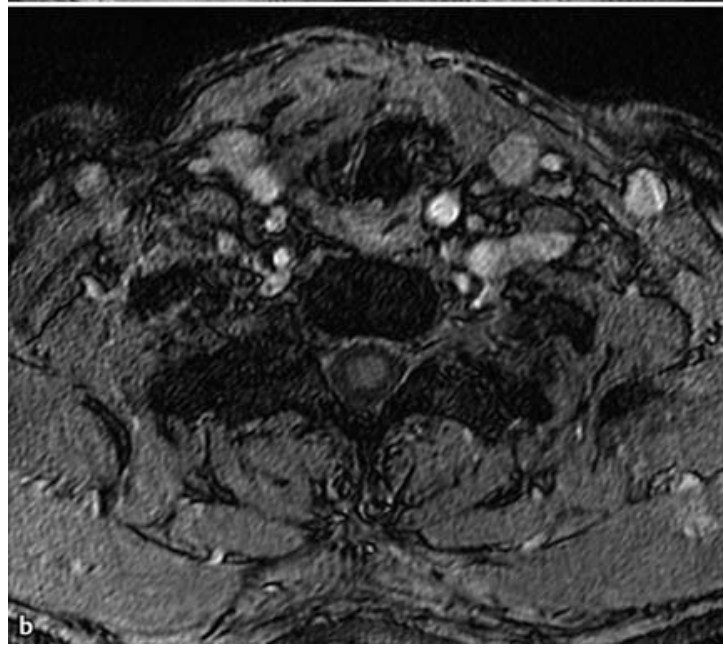
1. Ordnung treten diese Ränder nicht nur auf einer Seite, sondern an allen Grenzflächen auf. 
wenn sich diese zufällig innerhalb der Schichtlücke befinden.

Ein zweites Verfahren zur Elimination des Artefakts besteht darin, die Schichten nicht in der Reihenfolge ihrer Schichtposition anzuregen, sondern zunächst die ungeradzahligen, dann die geradzahligen. Hierdurch erreicht man einen maximalen zeitlichen Abstand bei der HF-Anregung zweier benachbarter Schichten.

Durch Kombination beider Methoden kann der Crosstalk auch mit sehr kleinen Schichtlücken (5-10\% der Schichtdicke) fast vollständig vermieden werden.

\section{Dephasierungsartefakt}

\section{- Ursache des Artefakts}

Dephasierungsartefakte treten dort auf, wo Spins, die sich innerhalb desselben Voxels befinden, zwischen Anregungspuls und Datenauslese unterschiedliche Magnetfelder erfahren. Dies ist vor allem bei intravaskulären Spins der Fall und wirkt sich bei der MR-Angiografie negativ aus: In Regionen mit turbulentem Fluss, z. B. hinter Stenosen oder in Aneurysmen, können Spins, die während der Auslese benachbart sind, unterschiedliche Gradientenfelder durchlaufen haben und daher unterschiedliche Phasen aufweisen. Weniger augenfällig, aber genauso störend ist das Artefakt an den Rändern von Blutgefäßen: Da der Geschwindigkeitsgradient aufgrund des paraboloiden Flussprofils bei laminarem Fluss vor allem zum Gefäßrand hin am größten ist, weisen auch hier Spins im selben Voxel unterschiedliche Phasen auf. Dies führt dazu, dass kleine Blutgefäße, deren Durchmesser jedoch größer als die nominelle räumliche Auflösung ist, nicht oder nicht durchgängig dargestellt werden können.

\section{Vermeidung des Artefakts}

Grundsätzlich kann man 2 Strategien anwenden, um Intravoxel-Dephasierungseffekte zu reduzieren:

- Man reduziert die Echozeit und gibt den Spins damit weniger Zeit zu dephasieren. Es ist grundsätzlich sinnvoll, eine möglichst hohe Auslesebandbreite zu verwenden.

- Man vergrößert die Ortsauflösung, d.h. reduziert die Voxelgröße, und bewirkt damit, dass alle Spins des Voxels eine möglichst homogene Phase haben.

Zusätzlich wirken sich Dephasierungseffekte in der kontrastmittelverstärkten MR-Angiografie weniger aus als bei den konventionellen Techniken wie Time-ofFlight- und Phasenkontrast-MR-Angiografie, da der
Kontrast bei der kontrastmittelverstärkten MR-Angiografie vor allem auf T1-Effekten und weniger auf Flusseffekten beruht.

\section{Dielektrischer Effekt}

\section{- Ursache des Artefakts}

Je höher die in der MRT verwendete Magnetfeldstärke ist, umso kleiner wird die für die Hochfrequenzanregung benötigte Wellenlänge. Ab einer Feldstärke von 3 Tesla liegen die HF-Wellenlänge und die untersuchten Objekte aufgrund der hohen dielektrischen Konstante von Gewebe in vergleichbarer Größenordnung. Hierdurch können sich innerhalb des untersuchten Objekts stehende Wellen ausbilden. Diese stehenden Wellen wiederum bewirken, dass die Hochfrequenzanregung nicht in jedem Voxel gleich stark ist, sondern Maxima und Minima bildet. Hierdurch erhält man an Hochfeld MR-Tomografen inhomogene Signalprofile.

\section{- Vermeidung des Artefakts}

Es gibt verschiedene Ansätze, dieses Artefakt zu minimieren: Einerseits kann man spezielle Pulssequenzen verwenden, die weniger sensitiv auf Inhomogenitäten des Hochfrequenzfelds sind [8], oder bei den Anregungspulsen solche Pulsformen auswählen, die ein möglichst homogenes Hochfrequenzfeld erzeugen [9]. Andererseits eröffnet sich ein Weg über die Hardware: Durch Verwendung von Mehrkanal-Sende- und Empfangsspulen können dielektrische Effekte eliminiert werden. Solche Spulen werden derzeit für 7-TeslaGeräte entwickelt, bei denen dielektrische Effekte die Bildqualität noch stärker stören als bei 3-T-Geräten; es ist zu erwarten, dass dieses Konzept auch in die Bildgebung bei 3 Tesla einfließt.

\section{Einfaltungsartefakt}

\section{- Ursache des Artefakts}

Ein Einfaltungsartefakt tritt dort auf, wo das Field of View (FOV) kleiner ist als das untersuchte Objekt. Da die Ortscodierung in der MRT durch eine Modulation der Präzessionsfrequenz mittels Magnetfeldgradienten über das gesamte Objekt erzielt wird, ist eine eindeutige Erfassung dieser Modulation entweder direkt über die Frequenz oder indirekt über den Phasenwinkel essenziell. Präzessionsfrequenzen, die größer sind als die halbe Abtastfrequenz, können nicht mehr erfasst werden und werden fälschlicherweise als niedrige Frequenz codiert. Da die Abtastfrequenz durch die Größe des FOV bestimmt wird, werden Körperteile, die in 
einer Richtung über das FOV hinausragen, von der entgegengesetzten Seite wieder in das MR-Bild hineingefaltet. In der Ausleserichtung wird dies automatisch verhindert, indem eine Abtastfrequenz gewählt wird, die doppelt so hoch ist wie die maximal zu erwartende Modulationsfrequenz. Dies ist in Phasencodierrichtung jedoch nicht der Fall. Daher treten Einfaltungsartefakte immer in Phasencodierrichtung auf. Während die Einfaltungsartefakte bei 2D-Sequenzen leicht zu erkennen und zuzuordnen sind, ist das bei 3D-Sequenzen schwieriger: Hier können Signale in andere Schichten einfalten und die dortige Bildinformation stören (Abb. 7).

\section{- Vermeidung des Artefakts}

Es gibt verschiedene Wege, um Einfaltungsartefakte zu vermeiden, die jedoch auch Nachteile mit sich bringen können:

- Vergrößerung des FOV: Nachteilig ist die daraus resultierende geringere Ortsauflösung, sofern diese nicht ebenfalls angepasst wird.

- Wahl der Phasencodierrichtung in Richtung der kürzesten Achse des Körperteils: Hierdurch können sich jedoch andere Artefakte, beispielsweise Bewegungsoder Pulsationsartefakte, störend auf die Bildinformation auswirken.

- Erhöhung der Zahl der Phasencodierschritte (Oversampling): Hiermit ist jedoch eine Verlängerung der Messzeit verbunden.

- Einsatz dedizierter Spulensysteme, die nur einen bestimmten Teil des Körperbereichs ausleuchten: Ist dieser Ausleuchtungsbereich kleiner als das FOV in Phasencodierrichtung, wird kein Signal außerhalb des FOV erfasst. Einen vergleichbaren Effekt erhält man durch Absättigung der Spins außerhalb des FOV durch Vorsättigungspulse, die in geeigneter Weise positioniert werden.

- Wahl eines schicht(block)selektiven Anregungspulses bei 3D-Sequenzen: Hierdurch kann man Einfaltungsartefakte in der 2. Phasencodierrichtung vermeiden.

\section{EPI-Ghosting}

\section{- Ursache des Artefakts}

Bei EPI-Sequenzen müssen die Auslesegradienten sehr schnell auf hohe Gradientenstärken geschaltet und wieder abgeschaltet werden, um eine schnelle Abtastung der Zeilen im k-Raum zu ermöglichen. Dabei bilden sich im Scanner Wirbelströme aus, die dem Aufbau des Gradienten entgegenwirken. Dies wiederum bewirkt, dass das Signalmaximum nicht in der Mitte des

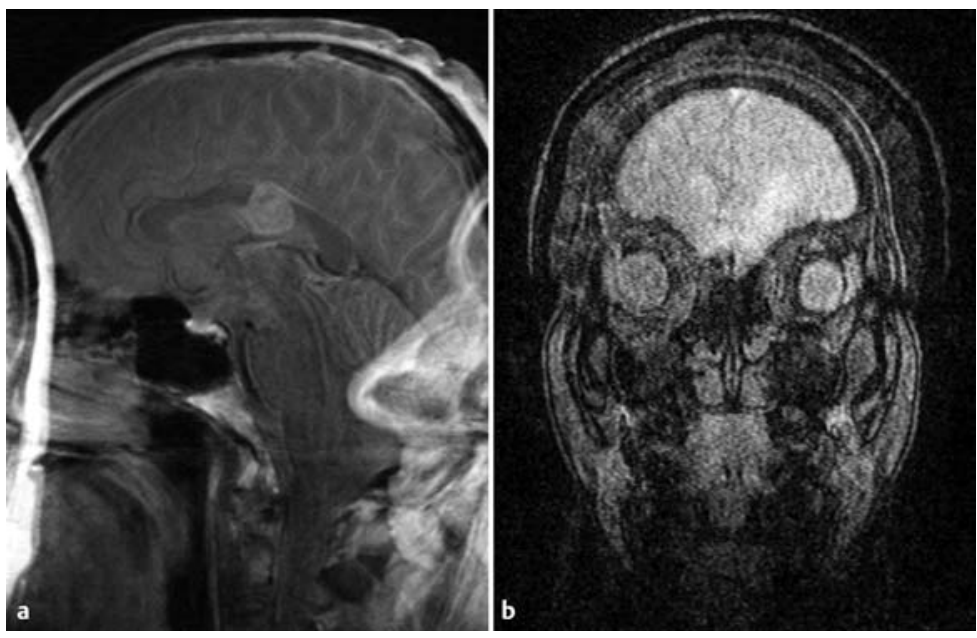

Abb. 7 Einfaltungsartefakte. a In Phasencodierrichtung (anterior - posterior) bei einer 2D-Sequenz. b In der 2. Phasencodierrichtung (anterior - posterior) bei einer 3D-Sequenz.

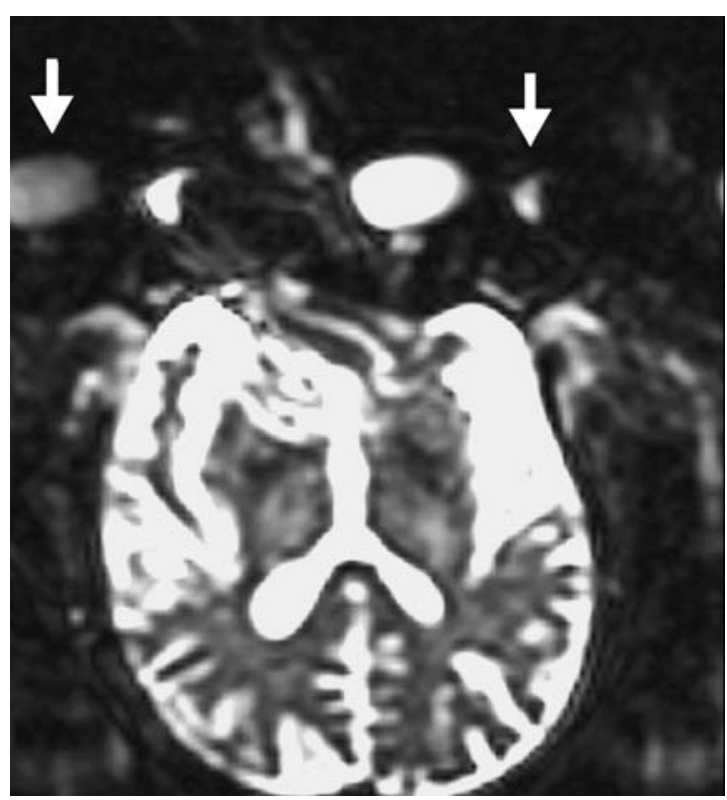

Abb. 8 EPI-Ghosting bei einer SpinEcho-EPI-Sequenz: Das abgebildete Objekt erscheint zusätzlich als Geisterbild mit einem Versatz von FOV/2 in Phasencodierrichtung (rechts links).

k-Raums registriert wird, sondern bei ungeraden Phasencodierschritten dahinter und bei geraden Phasencodierschritten davor. Aufgrund dieser Zickzack-Linie der Signalmaxima entstehen EPI-Geister, bei denen die Originalstruktur um FOV/2 in Phasencodierrichtung verschoben abgebildet wird (Abb. 8). Auch wenn die EPI-Geister ähnlich aussehen wie Einfaltungsartefakte, unterscheiden sie sich von ihnen dadurch, dass EPIGeister auch dann auftreten, wenn das untersuchte Objekt in Phasencodierrichtung kleiner ist als das FOV.

\section{- Vermeidung des Artefakts}

EPI-Ghosting kann vermieden werden, indem die Form der Gradientenpulse und der Zeitpunkt der Gradientenschaltung adjustiert werden (Preemphasis) [10]. Während dies früher von Hand eingestellt werden 


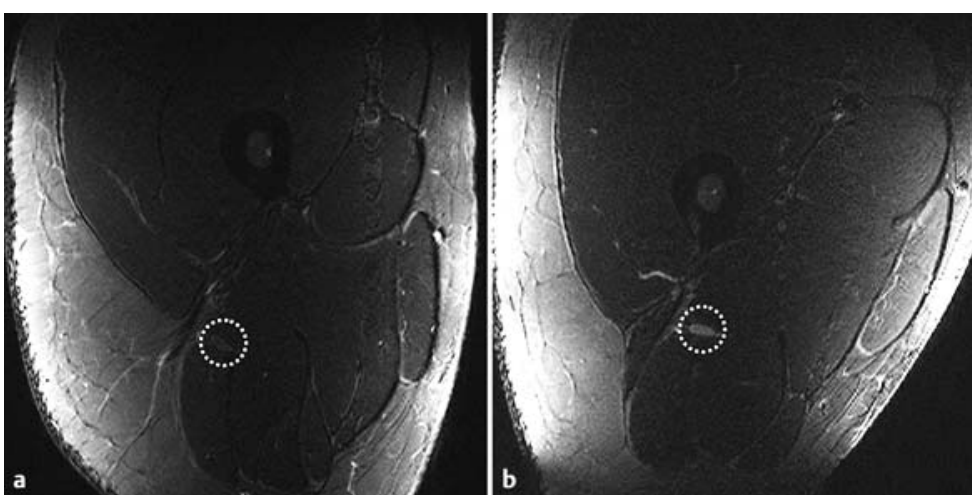

Abb. 9 Magic-Angle-Artefakt: transversale Schicht durch den Oberschenkel. a Ist der Oberschenkel parallel zum Magnetfeld orientiert, wird der N. ischiadicus (Kreis) isointens zum Muskel abgebildet. b Bei einer Neigung des Oberschenkels zum Magnetfeld von $55^{\circ}$ ist der N. ischiadicus deutlich hyperintens.

musste, werden Korrekturverfahren zur Beseitigung von EPI-Ghosting bei den neuen Scannergenerationen automatisch durchgeführt.

\section{Magic-Angle-Artefakt}

\section{- Ursache des Artefakts}

Magic-Angle-Artefakte treten ausschließlich in kollagenhaltigen Geweben wie Sehnen, Bändern und peripheren Nerven auf (Abb. 9). In diesen Strukturen hängt die T2-Zeit und damit das Signal in T2w Sequenzen davon ab, wie die Struktur in Relation zum statischen Magnetfeld orientiert ist. Dabei ergeben sich die niedrigsten T2-Zeiten, wenn die Struktur parallel zum Feld ausgerichtet ist, die höchsten T2-Zeiten werden dagegen gemessen, wenn die Struktur und das Magnetfeld einen Winkel von $55^{\circ}$ bzw. $125^{\circ}$ einschließen. Das Magic-Angle-Artefakt, das eine pathologische Signalerhöhung vortäuschen kann, ist darauf zurückzuführen, dass je nach Winkel die Dipol-Dipol-Interaktion zwischen Wasserspins und der quasikristallinen Struktur des Tropokollagens unterdrückt wird $[11,12]$. Er wird bei Sehnen und Bändern teilweise zur Bildgebung ausgenutzt, da diese Strukturen aufgrund ihrer äußerst geringen T2-Zeiten nur im Bereich der „magic angles“ von $55^{\circ}$ und $125^{\circ}$ dargestellt werden können.

\section{- Vermeidung des Artefakts}

Dieses Artefakt kann ausschließlich dadurch vermieden werden, dass die interessierende Struktur möglichst parallel zum Magnetfeld gelagert wird. Bei komplexen Strukturen, z. B. beim Plexus, ist dies jedoch nicht möglich. In solchen Fällen kann lediglich durch Wahl der Echozeit versucht werden, das Artefakt von Effekten pathologisch veränderter Strukturen sicher zu unterscheiden.

\section{Partialvolumeneffekt}

\section{- Ursache des Artefakts}

Dieses Artefakt tritt auf, wenn die räumliche Auflösung so gering ist, dass innerhalb eines Volumenelements (Voxel) mehrere Gewebeentitäten zu finden sind. In einem solchen Voxel ergibt sich die Signalintensität als gewichtetes Mittel der verschiedenen Signalintensitäten. In 2D-Sequenzen treten Partialvolumeneffekte vorwiegend in Schichtrichtung auf, da in solchen Sequenzen die räumliche Auflösung eine große Anisotropie aufweist. Zudem können die Spins unterschiedlicher Gewebe bei Gradienten-Echo- und Echo-PlanarImaging-Sequenzen in einem Voxel dephasieren, was eine fokale Signalreduktion zur Folge hat.

Generell können Partialvolumeneffekte dazu führen, dass kleine und schlecht kontrastierte Strukturen nicht mehr abgegrenzt werden können. Bei der Volumetrie führen Partialvolumeneffekte zu einer Unterschätzung des Läsionsvolumens. Vor allem in Gradienten-EchoSequenzen können darüber hinaus Strukturen vorgetäuscht werden, die anatomisch nicht vorhanden sind.

\section{- Vermeidung des Artefakts}

Um Partialvolumeneffekte zu minimieren, muss die räumliche Auflösung erhöht werden; bei 2D-Sequenzen gilt hierbei ein besonderes Augenmerk der Schichtdicke. Hierdurch wird jedoch das SNR verringert. Bei stark T2*w Sequenzen, vor allem bei Gradienten-Echo-EPI-Sequenzen, ist die Reduktion des SNR paradoxerweise jedoch geringer, als man es aus der Verkleinerung der Voxel schließen würde. Dies ist darauf zurückzuführen, dass die Intravoxeldephasierung und die damit verbundene Signalreduktion bei kleinen Voxeln geringer sind.

\section{Sättigungsartefakt}

\section{Ursache des Artefakts}

Werden auf Spins mit hoher Wiederholungsrate Hochfrequenzpulse eingestrahlt, kann die Longitudinalmagnetisierung nicht mehr ausreichend durch T1-Relaxation zwischen den Pulsen wiederhergestellt werden. Dabei kann die effektive Repetitionszeit (TR) kleiner sein als die für die Sequenz angegebene TR. Sind etwa Schichten oder Schichtblöcke gegeneinander anguliert und überlappen sich in bestimmten Bereichen, können in diesen überlappenden Bereichen Sättigungsartefakte auftreten. 
In der TOF-MR-Angiografie (TOF = Time-of-Flight) treten sehr häufig Sättigungsartefakte auf, da hier zur Unterdrückung des stationären Gewebes sehr kurze TR verwendet werden. So treten z.B. Artefakte auf, wenn das Gefäß über einen größeren Bereich innerhalb einer Schicht verläuft. Sie können auch auftreten, wenn die Dicke des Schichtblocks (S) bei 3D-TOF-Sequenzen so groß ist, dass die Spins viele Repetitionszeiten (TR) benötigen, um den gesamten Schichtblock zu durchqueren. In diesem Fall ist $\mathrm{TR}<<\mathrm{S} / \mathrm{v}_{\text {Blut }}$, wobei $\mathrm{v}_{\text {Blut }}$ die Fließgeschwindigkeit des Bluts ist. Bei der 2D-TOF können Sättigungsartefakte entstehen, wenn die Reihenfolge, in der die Schichten angeregt bzw. ausgelesen werden, mit der Richtung des Flusses übereinstimmt.

In den genannten Fällen entsteht eine Absättigung der Spins im Blut und somit ein Kontrastverlust zwischen Gefäßen und stationärem Gewebe.

\section{- Vermeidung des Artefakts}

Grundsätzlich muss bei mehreren, gegeneinander verkippten Schichten darauf geachtet werden, dass sich die Schichten nicht überschneiden oder die Akquisition nicht im Mehrschichtmodus erfolgt.

Um Sättigungsartefakte bei der TOF-MR-Angiografie zu vermeiden, sollte der Schichtblock in der interessierenden Region immer senkrecht zum Gefäß positioniert werden. Wenn die Gefäße in einem großen Volumen abgebildet werden sollen, ist es sinnvoll, anstatt eines dicken Schichtblocks mehrere, teilweise überlappende Schichtblöcke zu verwenden [13]. Ein weiteres Verfahren zur Reduktion der Sättigungsartefakte in dickeren Schichten oder bei langsamem Fluss ist die TONE-Technik (TONE $=$ „tilted optimized nonsaturating excitation“): Hier wird der Flip-Winkel des Hochfrequenzpulses linear vom proximalen zum distalen Ende des 3D-Blocks erhöht, um einen gleichmäßigen Kontrast zwischen Gefäß und Hintergrundgewebe über den gesamten 3D-Block zu garantieren [14].

\section{Spike}

\section{- Ursache des Artefakts}

Spikes treten auf, wenn es in einer der für Empfang und Verstärkung des MR-Signals verantwortlichen Hardwarekomponenten zu einer elektrischen Entladung kommt. Im k-Raum machen sich solche Entladungen als einzelne helle Punkte bemerkbar. Nach der Bildrekonstruktion ergeben die Spikes dann ein Streifenmuster über das gesamte MR-Bild, dessen Frequenz und Richtung davon abhängt, wo der Spike im k-Raum aufgetreten ist.

\section{- Vermeidung des Artefakts}

Sind diese Artefakte auf defekte Komponenten auf der Empfangsseite des MR-Tomografen zurückzuführen, müssen diese Komponenten ausgetauscht werden. Häufig treten Spikes jedoch auf, wenn die Luftfeuchtigkeit im Scannerraum zu gering ist, was den Aufbau elektrostatischer Felder mit anschließender Entladung begünstigt. Daher sollte unbedingt darauf geachtet werden, dass die vom Hersteller vorgegebenen Spezifikationen für die Luftfeuchtigkeit erfüllt werden.

\section{Stimulated-Echo-Artefakt}

\section{- Ursache des Artefakts}

Stimulated-Echo-Artefakte werden durch periodisch applizierte Hochfrequenzpulse verursacht, die vor allem in TSE-Sequenzen zu finden sind. Die StimulatedEchos überlagern sich mit den Spin-Echos und sind im MR-Bild meist als eine lokale Ansammlung feiner Linien zu erkennen. Das Artefakt kann ebenfalls durch Crosstalk der Hochfrequenzpulse benachbarter Schichten entstehen.

\section{- Vermeidung des Artefakts}

Ist das Artefakt durch Crosstalk entstanden, kann er beseitigt werden, indem der Schichtabstand erhöht oder die Schichten verzahnt angeregt werden. Ist das Artefakt dagegen durch eine Serie von $180^{\circ}$-Pulsen entstanden, kann er durch eine höhere Bandbreite oder durch eine Veränderung der Echozeit minimiert werden. Zur Vermeidung der Stimulated-Echo-Artefakte verwenden die meisten Hersteller Crusher-Gradienten, um die Transversalmagnetisierung nach jedem ausgelesenen Echo zu vernichten.

\section{Suszeptibilitätsartefakt}

\section{Ursache des Artefakts}

Suszeptiblitätsartefakte treten überall dort auf, wo das statische Magnetfeld nicht homogen ist. Im regulären Betrieb rühren solche Artefakte nicht vom MR-Tomografen selbst her, da der Hersteller ein Mindestmaß an Homogenität in einem definierten Radius garantieren muss. Jedes in den Scanner eingebrachte Objekt verursacht jedoch aufgrund seiner magnetischen Suszeptibilität geringfügige Veränderungen des Magnetfelds. An Grenzflächen zwischen unterschiedlichen Materialien, z. B. an Knochen-Luft-, Knochen-Gewebe-Grenzen 
Abb. 10 Suszeptibilitätsartefakt durch metallischen Inlay. a In direkter Nähe zum Metall wird das Signal komplett ausgelöscht (Pfeile).

b In entfernteren Schichten sind sowohl Signalauslöschungen als auch Hyperintensitäten zu sehen (Pfeile).
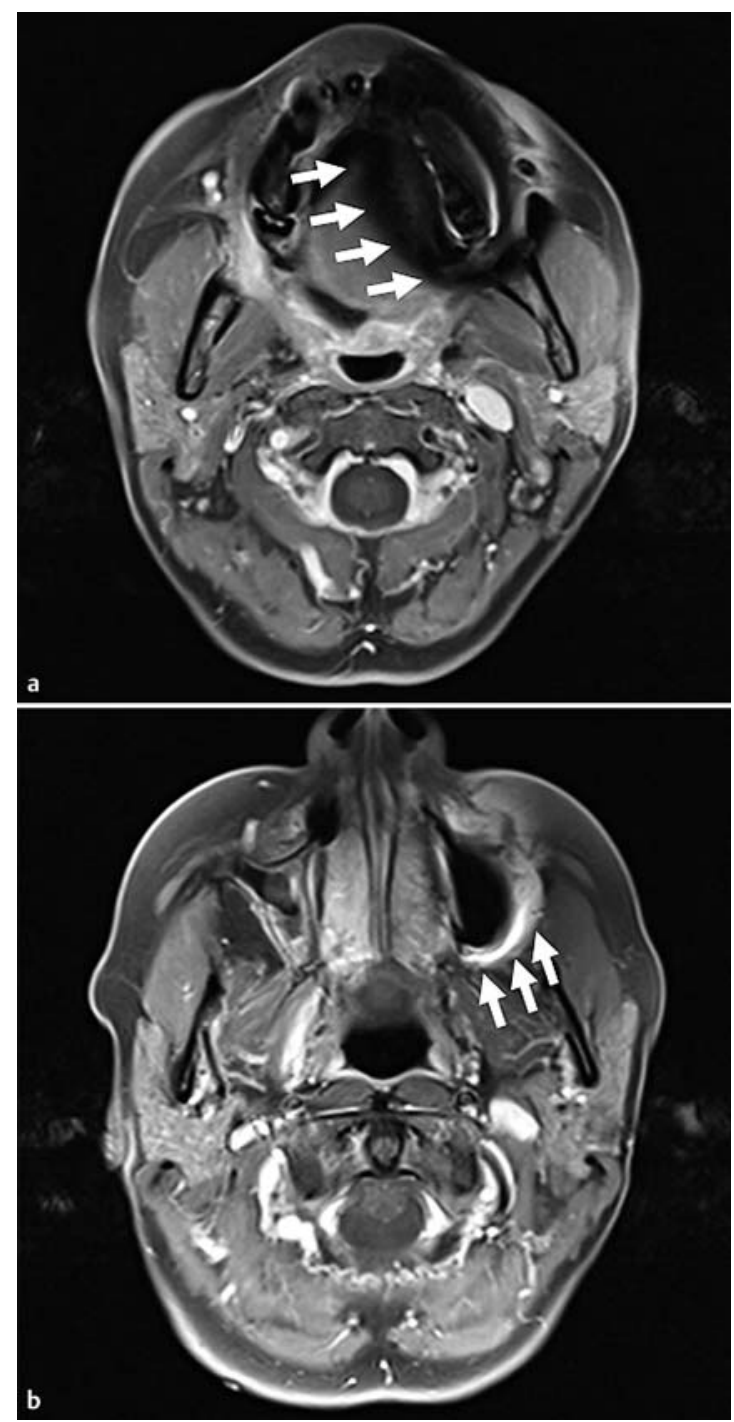

oder an metallischen Objekten, treten Suszeptibilitätssprünge auf, die zu lokalen Feldverzerrungen führen.

Diese Feldverzerrungen führen dazu, dass die Spins innerhalb eines Voxels unterschiedlich starke Felder ,sehen“. Während die daraus resultierende Dephasierung bei Spin-Echo-Sequenzen mittels Refokussierungspulsen kompensiert werden kann, ist dies bei GradientenEcho-Sequenzen nicht möglich und resultiert in einer lokalen Signalreduktion (Abb. 10). Besonders stark wirken sich die Artefakte bei Gradienten-Echo-EPISequenzen aus, da diese Sequenzen aufgrund der Vielzahl der verwendeten Gradienten-Echos und der langen effektiven Echozeit sehr empfindlich auf Feldverzerrungen sind. Außerdem sind die Artefakte umso größer, je höher die Feldstärke des Magneten ist.

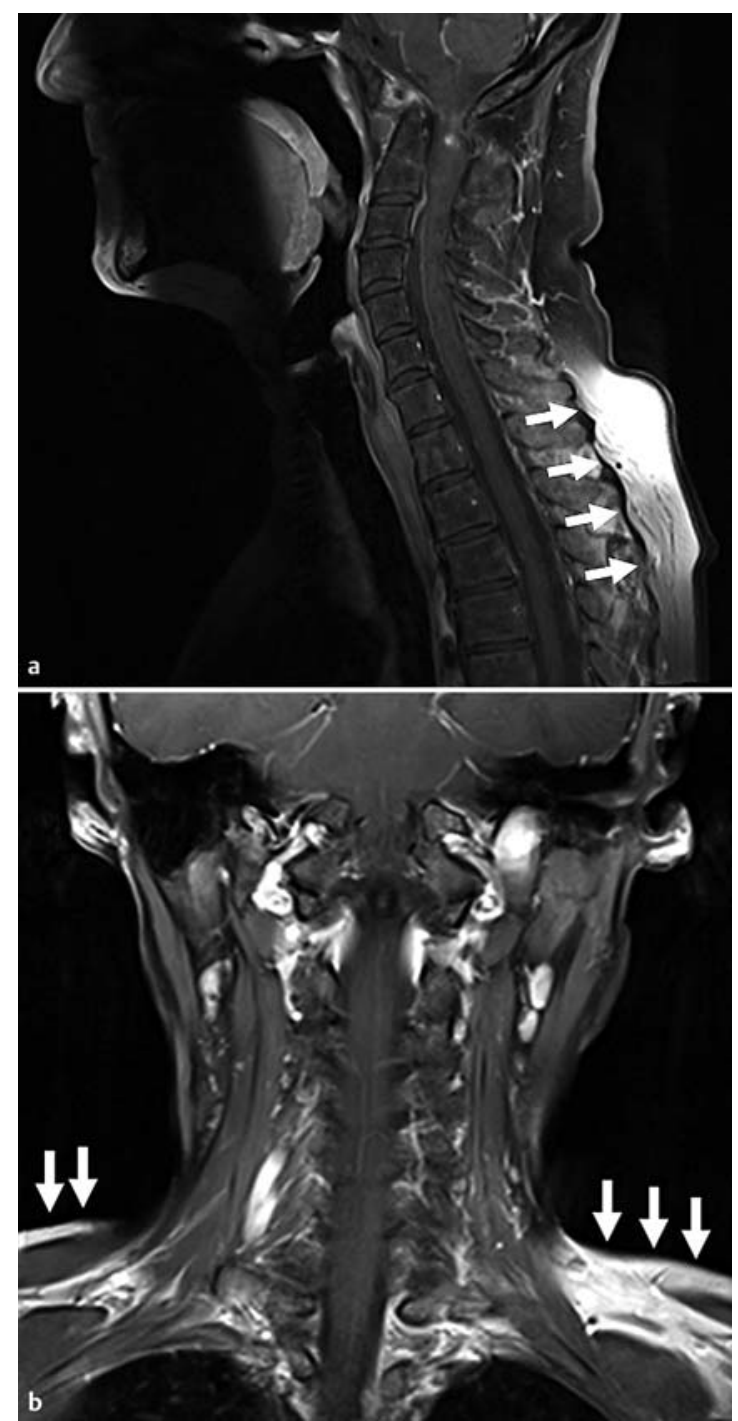

Abb. 11 Inhomogene spektrale Fettsättigung. Aufgrund starker Suszeptibilitätsänderungen zwischen Kopf, Hals und Schulter hat Fett in diesen Bereichen unterschiedliche Resonanzfrequenzen. Daher wird das Fettsignal von Kopf und Hals mit spektraler Fettsättigung unterdrückt, während die spektrale Fettsättigung auf Höhe der Schultern versagt (Pfeile). a Sagittale Aufnahmen. b Koronale Aufnahmen.

Aufgrund von Suszeptibilitätssprüngen treten bei spektraler Fettsättigung außerdem Artefakte auf. Diese sind darauf zurückzuführen, dass die Fettprotonen nicht in allen Regionen adäquat gesättigt werden können, da ihre Resonanzfrequenz im untersuchten Volumen nicht homogen ist (Abb. 11).

\section{- Vermeidung des Artefakts}

Sind die Artefakte sehr störend, beispielsweise in der Nähe von metallischen Implantaten, empfiehlt sich die Verwendung von Spin-Echo- anstelle von GradientenEcho-Sequenzen. Da es sich um Dephasierungsartefakte handelt, ist das Artefakt umso geringer, je kleiner die 
Echozeit ist. Die Verwendung einer hohen Auslesebandbreite ist außerdem von Nutzen; diese resultiert allerdings in einer Reduktion des SNR.

Bei EPI-Sequenzen, die besonders von Suszeptibilitätsartefakten betroffen sind, erreicht man eine geringere Sensitivität auf die Artefakte, wenn man den Echozug verkürzt. Dies kann durch den Einsatz von Parallel Imaging sowie durch Erhöhung der Auslesebandbreite erfolgen.

Die Artefakte aufgrund inhomogener Fettsättigung können durch Verwendung von Inversion-RecoveryFettsättigung anstelle von spektraler Fettsättigung beseitigt werden, denn die T1-Relaxationszeit, auf der die Inversion-Recovery-Fettsättigung beruht, wird durch die Feldinhomogenitäten nicht signifikant beeinflusst [15].

\section{Trunkationsartefakt (Gibbs-Ringing)}

\section{- Ursache des Artefakts}

Dieses Artefakt tritt vor allem an Grenzflächen von Geweben auf, an denen sich die Signalintensität sehr stark verändert. Solche scharfen Kanten resultieren im Frequenzraum (k-Raum) in hohen Signalamplituden bei hohen räumlichen Frequenzen. Wegen der begrenzten Auflösung der MRT wird jedoch bei der Signalaufnahme immer ein bestimmter Anteil von hohen Frequenzen abgeschnitten. Dies resultiert in einer periodischen Intensitätsschwankung um diese Kanten herum (Abb. 12), die umso stärker ist, je niedriger die räumliche Auflösung ist [16]. Daher tritt dieses Artefakt heute vor allem bei funktionellen und dynamischen MR-Verfahren auf, bei denen die räumliche Auflösung wie z. B. bei der Verwendung von EPI-Sequenzen stark begrenzt ist.

\section{- Vermeidung des Artefakts}

Dieses Artefakt kann in der MRT nie vollständig vermieden werden, da die Zahl der Pixel immer endlich ist. Allerdings kann er mit den routinemäßig verwendeten Sequenzen durch Erhöhung der Ortsauflösung so weit verringert werden, dass er im Bild kaum mehr zu erkennen ist.

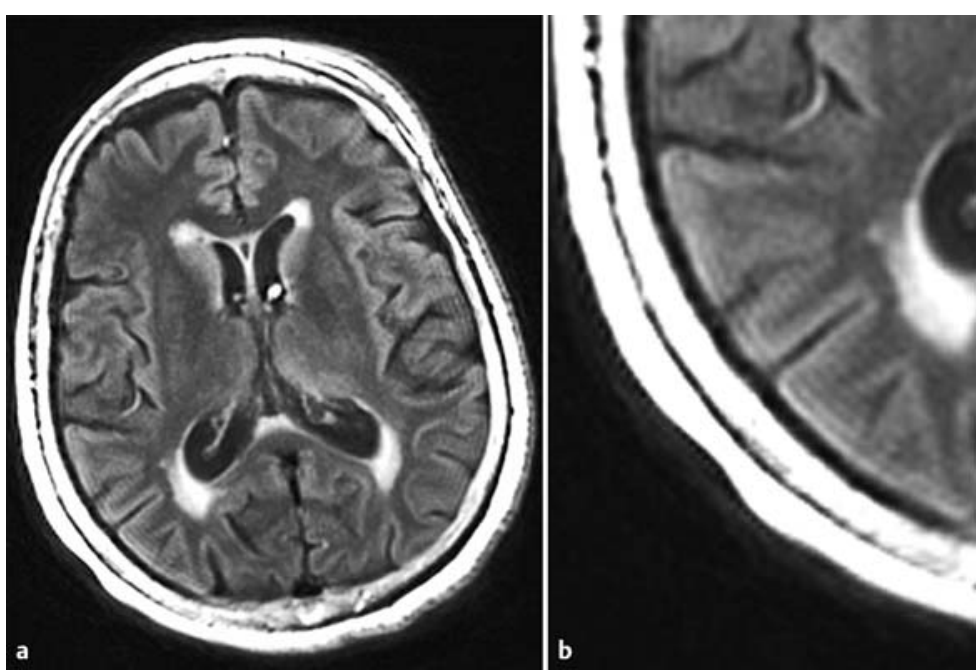

Abb. 12 Trunkationsartefakte (Gibbs-Ringing). a Transversale TSE-FLAIR-Aufnahme. b Ausschnitt aus a. Im Bildausschnitt sind deutlich die Oszillationen des Signals zu erkennen, die parallel zu der Signalkante verlaufen, die die Trunkationsartefakte auslöst.

\section{Verzeichnungsartefakt}

\section{- Ursache des Artefakts}

Bei Verzeichnungsartefakten tritt eine fehlerhafte räumliche Zuordnung des gemessenen Signals im MR-Bild auf. Es gibt 2 mögliche Ursachen für Verzeichnungsartefakte:

- Durch Nichtlinearität der Magnetfeldgradienten werden die Signale der betroffenen Volumenelemente fehlerhaft frequenz- oder phasencodiert. Solche Verzeichnungsartefakte treten mit zunehmendem Abstand vom Isozentrum des Magneten verstärkt auf.

- Lokale Feldinhomogenitäten entstehen z.B. aufgrund metallischer Objekte oder aufgrund von Grenzflächen zwischen Luft und Gewebe oder Luft und Knochen.

Diese Abweichungen des tatsächlichen Magnetfelds vom nominellen statischen Magnetfeld addieren sich auf die Gradientenfelder und führen zu einer fehlerhaften Registrierung des Signals. Aus diesen Gründen treten Verzeichnungsartefakte vor allem bei Verfahren der Datenauslese auf, bei denen sich Phasenfehler über eine längere Auslesezeit aufsummieren, so z. B. bei Gradienten-Echo-Sequenzen mit niedriger Auslesebandbreite oder bei EPI. EPI besitzt zwar in Frequenzcodierrichtung eine sehr hohe Bandbreite, allerdings ist die effektive Bandbreite in Phasencodierrichtung sehr niedrig, da aufgrund des Abtastverfahrens viel Zeit zwischen der Auslese der ersten und der letzten Phasencodierzeile verstreicht. 


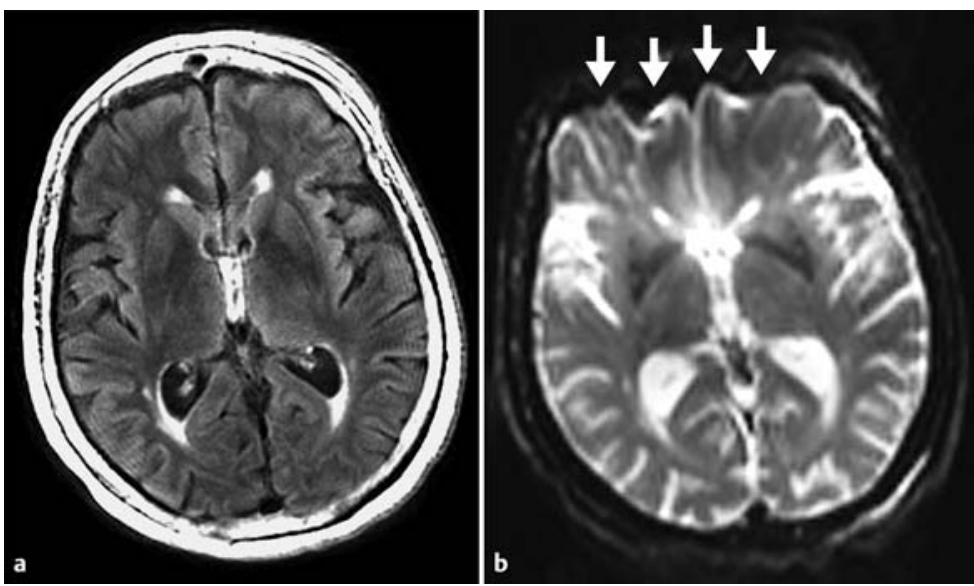

Abb. 13 Verzeichnungsartefakte. a TSE-FLAIR-Aufnahme. b Bei der Spin-Echo-EPI-Aufnahme sind frontal deutliche Verzeichnungen zu sehen, die auf die Magnetfeldinhomogenitäten im Bereich der Stirnhöhlen zurückzuführen sind.

\section{vermeidung des Artefakts}

Je nachdem welche der o.g. Ursachen vorliegt, gibt es unterschiedliche Ansätze zur Reduktion des Artefakts:

- Nicht lineare Gradienten: A posteriori kann die Verzeichnung korrigiert werden, da man die Linearität der Gradienten für jeden Punkt im Scanner erfassen kann und sich diese nicht über die Zeit verändert. Solche Verzeichnungskorrekturen existieren sowohl in der Schichtebene (2D) als auch im gesamten Volumen (3D) [17].

- Inhomogenes statisches Magnetfeld: Eine A-posteriori-Registrierung auf einen Vergleichsdatensatz ist unbefriedigend, da dieser Korrekturansatz nicht an der Ursache des Artefakts anpackt. Eine Alternative zu diesen Registrierungsverfahren ist eine gesonderte Aufnahme des Phasenfehlers mit anschließender Korrektur [18], die allerdings die Messzeit deutlich verlängert. Eine höhere Zeiteffizienz weist dagegen das B0-Field-Mapping auf, bei dem das Magnetfeld mit einer speziellen Gradienten-Multiecho-Sequenz Bildpunkt für Bildpunkt gemessen wird [19,20]. Basierend auf diesen Feldkarten kann dann auf den Phasenfehler rückgerechnet werden.
Einen gänzlich anderen Ansatz verfolgt der Einsatz des Parallel Imaging: Durch diese Technik kann der Echozug z. B. bei EPI drastisch verkürzt werden, sodass sich Phasenfehler nur noch in vermindertem Maß ausbilden können. Da man bei diesem Verfahren keine zusätzliche Messzeit benötigt, ist es inzwischen das führende Verfahren zur Verringerung der Verzeichnungsartefakte bei EPI (Abb. 13).

\section{Zipper}

\section{- Ursache des Artefakts}

Bildauslese in der MRT bedeutet, dass hochfrequente elektromagnetische Felder erfasst werden, die von den angeregten Spins ausgestrahlt werden. Damit dieses Signal nicht durch äußere hochfrequente Sender gestört wird, befindet sich der MR-Tomograf in einem gegenüber HF-Wellen abgedichteten Raum (FaradayKäfig). Durch Undichtigkeiten und Defekte in diesem Faraday-Käfig sowie durch HF-Quellen innerhalb des Scannerraums kann es dennoch zu einer Einstrahlung von HF-Signalen kommen. Wenn die Bandbreite des interferierenden MR-Signals klein ist, treten die Artefakte als helle Linie mit oszillierender Signalintensität in Phasencodierrichtung auf.

\section{- Vermeidung des Artefakts}

Tritt dieses Artefakt auf, sollte überprüft werden, ob die Tür zum Scannerraum offen ist oder ggf. elektrische Kabel ohne Führung über die Filterplatte in den Scannerraum verlegt sind. Beides muss vermieden werden, da sich durch offene Türen bzw. an elektrischen Leitern Störsignale in den Scannerraum ausbreiten können. Außerdem muss untersucht werden, ob es ggf. im Scannerraum selbst Störquellen gibt, z. B. flackernde Glühbirnen. Ist all dies nicht der Fall, dann liegt mit hoher Wahrscheinlichkeit ein Defekt des FaradayKäfigs vor, der nur vom Hersteller diagnostiziert und beseitigt werden kann. 


\section{Kernaussagen}

- Die MRT ist im Vergleich zu anderen radiologischen Verfahren deutlich empfindlicher, was Bildartefakte angeht. Das Bildsignal hängt von vielen physiologischen und physikalischen Parametern des Gewebes ab.

- Artefakte können sowohl pathologische Befunde maskieren als auch krankhafte Veränderungen vortäuschen. Daher ist es wichtig, die Artefaktmuster zu (er)kennen und zu wissen, wie man sie vermeidet bzw. minimiert.

- Dephasierungs-, Suszeptibilitäts-, Magic-Angle-, Bewegungs- und Verzeichnungsartefakte sind vor allem vom Patienten abhängig bzw. von der Region, die aufgenommen wird. Allerdings haben auch Sequenzeinstellungen Einfluss auf diese Artefakte.
- Sättigungs-, Chemical-Shift-, Stimulated-Echo-, Trunkations- und Einfaltungsartefakte sowie Crosstalk, Blurring und Partialvolumeneffekt sind vor allem von der verwendeten Sequenz bzw. den eingestellten Sequenzparametern abhängig.

- Die Hardware kann dielektrische Effekte, Spikes und Zipper verursachen.

\section{Abstract}

Magnetic Resonance Imaging (MRI) offers a variety of contrasts which can be modified by choosing the appropriate sequence parameters. Several tissue parameters and physiological variables influence signal intensity, as relaxation times, spin density, diffusion, oxygenation of blood or blood flow. This multiparametric dependency of MRI accounts for its excellent soft tissue contrast, but is also the main cause for its high sensitivity to artifacts.

This article provides a description of the most important MR artifacts. We focus particularly on the physical and technical principles of the artifacts and on possible workarounds and techniques to allow for artifact-free MR images.

\section{Key words}

Magnetic resonance imaging · artifacts - motion • susceptibility · dephasing · fast imaging techniques

\section{Über die Autorin}

\section{Sabine Heiland}

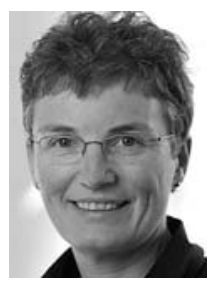

Jahrgang 1965. Studium der Physik an den Universitäten Saarbrücken und Heidelberg. Promotion 1993. 1993 2004 Wissenschaftliche Mitarbeiterin in der Abteilung Neuroradiologie des Universitätsklinikums Heidelberg. 1997 Fachanerkennung für Medizinphysik (DGMP). 2001 Habilitation zum Thema „Entwicklung MR-tomographischer Methoden zur Erfassung funktioneller Parameter in der radiologischen Diagnostik von Krankheiten des Zentralnervensystems“. Seit 2004 Professorin für Experimentelle Neuroradiologie und Leiterin der Sektion Experimentelle Radiologie am Universitätsklinikum Heidelberg.

\section{Korrespondenzadresse}

Prof. Dr. Sabine Heiland

Universitätsklinikum Heidelberg

Sektion Experimentelle Radiologie

Abteilung Neuroradiologie

Im Neuenheimer Feld 400

69120 Heidelberg

Tel. 06221 56-7566

Fax: 06221 56-4673

E-Mail: sabine.heiland@med.uni-heidelberg.de 


\section{Literatur}

1 Arena L, Morehouse HT, Safir J. MR imaging artifacts that simulate disease: How to recognize and to eliminate them. Radiographics 1995; 15: 1373 - 1394

2 Ehman RL, Felmlee JP. Flow artifact reduction in MRI: a review of the roles of gradient moment nulling and spatial presaturation. Magn Reson Med 1990; 14: 293-307

3 Ordidge RJ, Helpern JA, Qing ZX et al. Correction of motional artifacts in diffusion-weighted MR images using navigator echoes. Magn Reson Imaging 1994; 12: 455 - 460

4 Pipe JG. Motion correction with PROPELLER MRI: application to head motion and free-breathing cardiac imaging. Magn Reson Med 1999; 42: 963 - 969

5 Smith AS, Weinstein MA, Hurst GC et al. Intracranial chemicalshift artifacts on MR images of the brain: observations and relation to sampling bandwidth. AJR Am J Roentgenol 1990; 154: $1275-1283$

6 Savci G, Yazici Z, Sahin N et al. Value of chemical shift subtraction MRI in characterization of adrenal masses. AJR Am J Roentgenol 2006; 186: 130-135

7 Venkataraman S, Braga L, Semelka RC. Imaging the fatty liver. Magn Reson Imaging Clin N Am 2002; 10: 93-103

8 Collins CM, Liu W, Schreiber W et al. Central brightening due to constructive interference with, without, and despite dielectric resonance. J Magn Reson Imaging 2005; 21: 192 - 196

9 Setsompop K, Wald LL, Alagappan V et al. Parallel RF transmission with eight channels at 3 Tesla. Magn Reson Med 2006; 56: $1163-1171$

10 Papadakis NG, Martin KM, Pickard JD et al. Gradient preemphasis calibration in diffusion-weighted echo-planar imaging. Magn Reson Med 2000; 44: 616-624
11 Bydder M, Rahal A, Fullerton GD, Bydder GM. The magic angle effect: a source of artifact, determinant of image contrast, and technique for imaging. J Magn Reson Imaging 2007; 25 (2): $290-300$

12 Stoll G, Bendszus M, Perez J, Pham M. Magnetic resonance imaging of the peripheral nervous system. J Neurol 2009; 256: $1043-1051$

13 Davis WL, Warnock SH, Harnsberger HR et al. Intracranial MRA: single volume vs. multiple thin slab 3D time-of-flight acquisition. J Comput Assist Tomogr 1993; 17: 15-21

14 Priatna A, Paschal CB. Variable-angle uniform signal excitation (VUSE) for three-dimensional time-of-flight MR angiography. J Magn Reson Imaging 1995; 5: 421 - 427

15 Delfaut EM, Beltran J, Johnson G et al. Fat suppression in MR imaging: techniques and pitfalls. Radiographics 1999; 19 : $373-382$

16 Czervionke LF, Czervionke JM, Daniels DL, Haughton VM. Characteristic features of MR truncation artifacts. AJR Am J Roentgenol 1988; 151: 1219-1228

17 Karger CP, Höss A, Bendl R et al. Accuracy of device-specific 2D and $3 \mathrm{D}$ image distortion correction algorithms for magnetic resonance imaging of the head provided by a manufacturer. Phys Med Biol 2006; 51: 253-261

18 Chen NK, Wyrwicz AM. Optimized distortion correction technique for echo planar imaging. Magn Reson Med 2001; 45: $525-528$

19 Jezzard P, Barnett AS, Pierpaoli C. Characterization of and correction for eddy current artifacts in echo planar diffusion imaging. Magn Reson Med 1998; 39: 801 - 812

20 Schmithorst VJ, Dardzinski BJ, Holland SK. Simultaneous correction of ghost and geometric distortion artifacts in EPI using a multiecho reference scan. IEEE Trans Med Imaging 2001; 20: $535-539$ 


\section{CME-Fragen}

Die folgenden Fragen beziehen sich auf den vorangehenden Beitrag. Bitte schicken Sie uns die entsprechenden Lösungsbuchstaben. Jeweils eine Antwort ist richtig. Die Vergabe von CME-Punkten ist an die korrekte Beantwortung der Multiple-Choice-Fragen gebunden.

\section{1}

Welche der folgenden Aussagen ist richtig? Einfaltungsartefakte treten auf,
A wenn das Objekt weit entfernt vom Isozentrum positioniert ist.
B wenn das Objekt in Frequenzcodierrichtung größer ist als das FOV.
C wenn das Objekt in Frequenzcodierrichtung kleiner ist als das FOV.
D wenn das Objekt in Phasencodierrichtung größer ist als das FOV.
E wenn das Objekt in Phasencodierrichtung kleiner ist als das FOV.

\section{2}

Wie können Einfaltungsartefakte entlang der Schichtcodierrichtung bei 3D-Sequenzen nicht vermieden werden?
A Oversampling in Schichtcodierrichtung
B Erhöhung der Auflösung in Schichtcodierrichtung
C Auswahl spezieller Hochfrequenzspulen, die selektiv den interessierenden Bereich ausleuchten
D Vergrößerung des Schichtblocks
E Wahl eines schichtselektiven Anregungspulses

3

Welche der folgenden Aussagen ist richtig? Um Artefakte aufgrund der chemischen Verschiebung zwischen Fett und Wasser zu vermeiden, kann man
A die T1-Gewichtung durch Verkürzung der Echozeit erhöhen.
B das Field of View verkleinern.
C die Auslesebandbreite erhöhen.
D die Repetitionszeit erhöhen.
E einen Magnetization-Transfer-Puls verwenden.

Welche der folgenden Aussagen ist nicht richtig?
A Bei der „opposed-phase“-Bedingung löschen sich die Signale der Wasser- und Fettprotonen ganz oder teilweise aus.

B Die kürzeste Echozeit, bei der bei 3,0 T eine „opposed-phase“-Bedingung auftritt, ist 2,2 ms.

C Die kürzeste Echozeit, bei der bei 1,5 T eine „opposed-phase“-Bedingung auftritt, ist 2,2 ms.

D Die kürzeste Echozeit, bei der bei 1,5 T eine „in-phase“-Bedingung auftritt, ist 4,4 ms.

E Bei der „in-phase“-Bedingung verstärken sich die Signale der Wasser- und Fettprotonen.

5

Welche der folgenden Aussagen ist nicht richtig?
A Falls 2 Schichtblöcke gegeneinander anguliert sind, kann es zu Sättigungsartefakten kommen.

B In der TOF-MR-Angiografie können Sättigungseffekte auftreten, wenn das Gefäß ganz oder teilweise innerhalb der Schicht verläuft.

C Bei der 2D TOF können Sättigungsartefakte auftreten, wenn die Reihenfolge, in der die Schichten angeregt bzw. ausgelesen werden, mit der Richtung des Flusses übereinstimmt.

D In der TOF-MR-Angiografie können Sättigungseffekte auftreten, falls TR $\gg S / v_{\text {Blut }}$, wobei S die Schichtdicke und $\mathrm{v}_{\text {Blut }}$ die Fließgeschwindigkeit des Bluts ist.

E In der TOF-MR-Angiografie können Sättigungseffekte auftreten, falls $T R \ll<S / v_{\text {Blut }}$, wobei S die Schichtdicke und $\mathrm{v}_{\text {Blut }}$ die Fließgeschwindigkeit des Bluts ist.

\section{6}

Was kann man tun, um Trunkationsartefakte zu reduzieren?
A die Echozeit verringern
B die Repetitionszeit erhöhen
C die Ortsauflösung verringern
D die Ortsauflösung erhöhen
E Oberflächenspulen einsetzen 


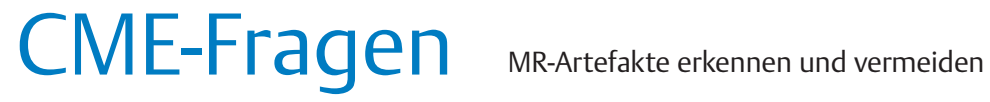

\section{7}

Welche der folgenden Aussagen ist nicht richtig?
A Suszeptibilitätsartefakte treten bei hohen Magnetfeldern zunehmend auf.

B Um Suszeptibilitätsartefakte zu minimieren, sollte man eine möglichst hohe Auslesebandbreite verwenden.

C Um Suszeptibilitätsartefakte zu minimieren, sollte man die Echozeit verringern.

D Suszeptibilitätsartefakte bei EPI-Sequenzen können durch den Einsatz von Parallel Imaging verringert werden.

E Suszeptibilitätsartefakte treten nur an metallischen Fremdkörpern auf.

\section{8}

Welche der folgenden Aussagen ist nicht richtig?
A Dephasierungsartefakte können in der TOF-MR-Angiografie in Regionen mit turbulentem Fluss auftreten.

B Dephasierungsartefakte können auch bei laminarem Fluss zu einer Unterschätzung des Gefäßlumens führen.

C Dephasierungsartefakte spielen in der MR-Angiografie vor allem bei Verwendung von Kontrastmitteln eine Rolle.

D Zur Reduktion von Dephasierungsartefakten sollte man die Echozeit möglichst klein wählen.

E Zur Reduktion von Dephasierungsartefakten sollte man eine möglichst hohe Bandbreite verwenden.

\section{9}

Welche der folgenden Aussagen ist richtig? Verzeichnungsartefakte treten vor allem bei EPI-Sequenzen auf, weil diese
A in Phasencodierrichtung eine extrem hohe Bandbreite aufweisen und es daher zur Summierung von Phasenfehlern kommt.
B in Phasencodierrichtung eine extrem niedrige Bandbreite aufweisen und es daher zur Summie- rung von Phasenfehlern kommt.
C in Frequenzcodierrichtung eine extrem hohe Bandbreite aufweisen und es daher zur Summierung von Phasenfehlern kommt.
D in Frequenzcodierrichtung eine extrem niedrige Bandbreite aufweisen und es daher zur Summie- rung von Phasenfehlern kommt.
E ausschließlich bei hohen Feldstärken verwendet werden können.

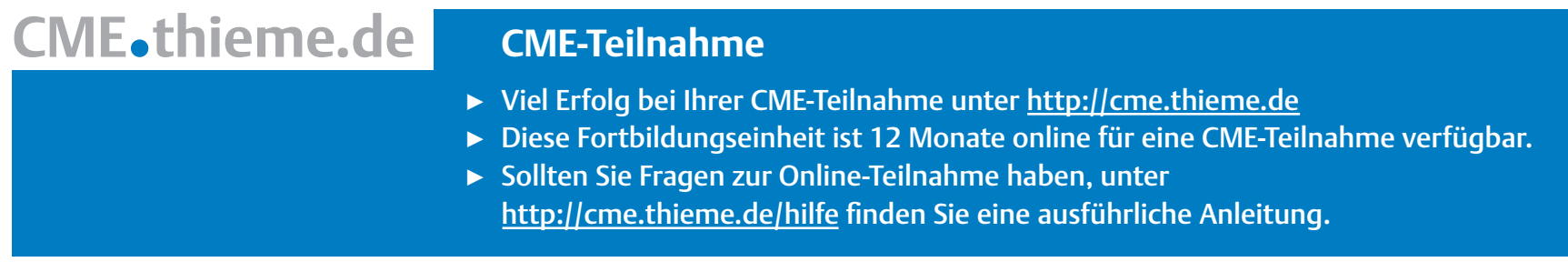

Welche der folgenden Aussagen ist richtig?
A Stimulated-Echo-Artefakte zeigen sich als durchgängiges Streifenmuster im gesamten MR-Bild.
B Spikes zeigen sich als einzelner heller Punkt im MR-Bild.
C Spikes zeigen sich als durchgängiges Streifenmuster im gesamten MR-Bild.
D Ein Zipper zeigt sich als einzelner heller Punkt im MR-Bild.
E Ein Zipper zeigt sich als durchgängiges Streifenmuster im gesamten MR-Bild. 